\title{
针对高维优化问题的快速追峰采样方法*
}

\author{
武宇飞 ${ }^{1}$ 龙 腾 ${ }^{1,2}$ 史人赫 ${ }^{1}$ WANG G Gary ${ }^{3}$
}

(1. 北京理工大学宇航学院 北京 100081;

2. 北京理工大学飞行器动力学与控制教育部重点实验室 北京 100081 ;

3. 西门菲莎大学机电工程学院 素里 V3T 0A3 加拿大)

摘要: 基于计算试验设计和代理模型的近似优化策略在现代复杂系统工程设计中得到了广泛应用, 其中追峰采样方法(Mode pursuing sampling, MPS)是一种代表性的近似优化策略。分析并针对 MPS 处理高维优化问题时效率低下的缺陷, 提出了基于 重点设计空间的快速追峰采样方法(RMPS-SDS), 将重点设计空间的思想引入 MPS 框架, 定制了一套样本点分配策略以增强 MPS 的局部搜索能力与收敛速度, 从而提高求解高维优化问题的效率。采用一系列标准数值测试问题和工程设计问题检验 RMPS-SDS 方法的性能, 并与 MPS 和 GA 进行了对比研究。研究结果表明, 在相同模型调用次数前提下, RMPS-SDS 的优 化结果更接近理论全局最优解, 且鲁棒性更好。与标准 MPS 相比, RMPS-SDS 方法求解高维优化问题的效率、收玫性和鲁 棒性都具有明显优势, 更具有工程实用性。

关键词: 追峰采样; 自适应代理模型; 近似优化; 重点设计空间; 全局优化

中图分类号: V221

\section{A Rapid Mode Pursuing Sampling Method for High Dimensional Optimization Problems}

\author{
WU Yufei $^{1} \quad$ LONG Teng $^{1,2}$ SHI Renhe ${ }^{1} \quad$ WANG G Gary ${ }^{3}$
}

(1. School of Aerospace Engineering, Beijing Institute of Technology, Beijing 100081;

2. Key Laboratory of Dynamics and Control of Flight Vehicle of Ministry of Education,

Beijing Institute of Technology, Beijing 100081;

3. School of Mechatronic Systems Engineering, Simon Fraser University, Surrey, BC V3T 0A3, Canada)

\begin{abstract}
Approximate optimization strategies using design of computer experiments (DoCE) and metamodels have been widely applied in design of modern complex engineering systems. Mode pursuing sampling method (MPS) is a representative of such optimization algorithms. A rapid mode pursuing sampling method using significant design space concept (notated as RMPS-SDS) is proposed in this work to alleviate the low efficiency problem of MPS in solving high dimensional optimization problems. The idea of significant design space is incorporated into the MPS framework, and a sample point allocation strategy is designed to enhance the local search capability and convergence speed of MPS. RMPS-SDS is tested on a number of standard numerical benchmark problems and two engineering design problems and compared with MPS and GA. The comparison results indicate that with the same computational budget (i.e., the same number of function evaluations), results of RMPS-SDS are much closer to the theoretical global optima with lower standard deviation for multiple runs. It is thus demonstrated that the proposed RMPS-SDS outperforms the standard MPS in terms of efficiency, convergence, and robustness in solving high dimensional optimization problems, which is more promising for engineering practices.
\end{abstract}

Key words: mode pursuing sampling; adaptive metamodel; approximate optimization; significant design space; global optimization

* 国家自然科学基金(51675047, 11372036)、航空科学基金(2015ZA72004)、 北京理工大学国际科技合作专项计划(GZ2018015101)、北京理工大学研 究生科技创新(2018CX10001)资助项目。20180321 收到初稿, 20181023 收到修改稿 


\section{0 前言}

复杂工程设计中应用优化设计技术可以改善设 计质量, 缩短设计周期。为了提高设计可信度, 计 算耗时的高精度分析模型在工程设计优化中的应用 日益广泛，例如结构有限元分析(Finite element analysis, FEA)、计算流体力学(Computational fluid dynamics, CFD)、计算电磁学(Computational electromagnetics, CEM)等。由于通过有限差分法等方式获得梯度需要 反复调用分析模型, 传统的基于梯度的局部搜索算法 (如增广拉格朗日乘子法、可行方向法、序列二次规划) 并不适用。对于基于概率的全局探索算法(如遗传算 法、模拟退火算法、粒子群算法), 分析模型调用次 数可达成千上万次, 使计算成本难以接受。为提高 计算效率, 基于代理模型的近似优化策略在工程优化 领域得到了深入研究与广泛应用 ${ }^{[1-2]}$ 。

代理模型的精度与原分析模型相当, 但计算成 本较低。目前使用最广泛的代理模型包括多项式响 应面(Polynomial response surface method, PRSM) ${ }^{[3]}$ 、 径向基函数(Radial basis function, $\mathrm{RBF})^{[4]}$ 、克里金模 型(Kriging, $\mathrm{KRG})^{[5]}$ 等。

文献[6]指出, 基于代理模型的近似优化策略可 分为静态近似优化策略和自适应近似优化策略。静 态近似优化策略要求代理模型在整个设计空间都有 较高的近似精度, 精度未达到要求需要更新代理模 型; 而自适应近似优化策略着重提高可能存在全局 最优点的区域的近似精度, 从而在降低采样数量要 求的同时引导优化过程快速收玫到全局最优解。自 适应近似优化策略的代理模型更新方式主要有基于 空间缩减序列采样和基于空间填充序列采样两种。

基于空间缩减序列采样方法主要包括: 信赖域 (Trust region)方法、分割平面(Cutting plane)方法、 重点设计空间(Significant design space, SDS)等。部 分代表性研究成果总结如下: 龙腾等 ${ }^{[7]}$ 提出了基于 信赖域的动态 RBF 代理模型优化策略(Optimization strategy using trust region based dynamic RBF optimization strategy, TR-DRBF); CHENG 等 ${ }^{[8]}$ 提出 了基于信赖域的追峰采样方法(Trust region based mode pursuing sampling, TRMPS); WANG 等 ${ }^{[9]}$ 提出了 采用分割平面的自适应响应面方法(Adaptive response surface method); 彭否等 ${ }^{[10,11]}$ 提出了重点设计空间的思 想, 并发展了一种序列 RBF 优化策略(Sequential RBF, $\mathrm{SRBF})$; 龙腾等 ${ }^{[12]}$ 在 SDS 方法的基础上, 提出了采用 智能空间探索策略, 进而发展了一种改进的高效自适 应响应面方法 (Efficient adaptive response surface method using intelligent space exploration strategy, ARSM-ISES)等。与信赖域方法和分割平面法相比, SDS 方法直接根据最优性与近似精度构造兴趣区域, 有效提高了代理模型在最优解附近的近似精度, 而且 算法参数更少 ${ }^{[6]}$ 。

具有代表性的基于空间填充序列采样方法包括: 高效全局优化方法 (Efficient global optimization, EGO) ${ }^{[13]}$ 、追峰采样方法(Mode pursuing sampling, MPS) ${ }^{[14]}$ 和基于模糊聚类的全局优化策略(Metamodelbased global optimization using fuzzy clustering for design space reduction, FCR $)^{[15]}$ 等。其中, MPS 算法 在处理低维问题时效率明显高于常见的全局优化算 法, 而且基于概率分布的有偏采样在理论上保证了 MPS 算法以概率 1 收敛到全局最优解 ${ }^{[14]}$ 。然而 DUAN 等 ${ }^{[16]}$ 指出, MPS 处理高维问题时存在效率较 低的缺陷。本文研究发现, MPS 求解高维问题效率较 低, 其主要原因是局部搜索能力不足, 可能连续多次 迭代都不能改善目标函数值, 浪费大量计算量。考虑 到基于空间缩减序列采样方法的近似策略具有良好 的局部搜索能力, 本文将重点设计空间方法与 MPS 算法相结合, 以提高其处理高维优化问题的效率。

本文提出的基于重点设计空间的快速追峰采样 方法(Rapid mode pursuing sampling method using significant design space concept, RMPS-SDS)通过一 种样本点分配策略, 将样本点分为全局探索样本点和 局部搜索样本点, 全局探索样本点沿用基于概率分布 的追峰采样机理, 而局部搜索样本点则取自构造的重 点设计空间内。采用基于 Maximin 准则的拉丁超方设 计进行初始和序列采样, 以提高样本点空间均布性。 最后通过标准测试算例上工程优化案例比较研究, 检 验RMPS-SDS 的性能优势。

\section{1 追峰采样方法简介}

\section{1 径向基函数简介}

径向基函数是一种常用的多变量空间插值型代 理模型方法, 其基本形式为

$$
\hat{f}_{R B F}(\boldsymbol{x})=\sum_{i=1}^{n_{s}} \boldsymbol{w}_{i} \phi\left(\left\|\boldsymbol{x}-\boldsymbol{x}_{i}\right\|\right)=\boldsymbol{w}^{\mathrm{T}} \boldsymbol{\phi}
$$

根据插值条件, 权重系数矢量 $\boldsymbol{w}$ 可以按式(2)求解

$$
\boldsymbol{A}=\left(\begin{array}{ccc}
\boldsymbol{w}=\boldsymbol{A}^{\mathrm{T}} \boldsymbol{y} \\
\phi\left(\left\|\boldsymbol{x}_{1}-\boldsymbol{x}_{1}\right\|\right) & \cdots & \phi\left(\left\|\boldsymbol{x}_{1}-\boldsymbol{x}_{n_{s}}\right\|\right) \\
\vdots & & \vdots \\
\phi\left(\left\|\boldsymbol{x}_{n_{s}}-\boldsymbol{x}_{1}\right\|\right) & \cdots & \phi\left(\left\|\boldsymbol{x}_{n_{s}}-\boldsymbol{x}_{n_{s}}\right\|\right)
\end{array}\right)
$$


本文中径向函数 $\phi(r, c)$ 取高斯函数

$$
\phi(r, c)=\exp \left(-c r^{2}\right)
$$

参数 $c$ 根据经验公式 ${ }^{[6]}$ 取为

$$
c=\left(\left(\max \left(x_{i}\right)-\min \left(x_{i}\right)\right) / n_{s}\right)^{1 / n_{v}}
$$

式中, $n_{v}$ 为设计变量 $\boldsymbol{x}$ 的维度, $n_{s}$ 为样本点个数。

\section{2 追峰采样方法}

MPS 算法初始样本点数为

$$
n_{0}=\frac{\left(n_{v}+1\right)\left(n_{v}+2\right)}{2}-1+n_{v}
$$

将初始样本点及其真实模型响应值加入高精度样本 点集 $\boldsymbol{Y}$, 用以构造 RBF 代理模型。在设计空间内生 成 $N$ (例如取 10000 ) 个简单样本点, 将它们及其 $\mathrm{RBF}$ 响应值加入简单样本点集 $\boldsymbol{X}$ 。对 $\boldsymbol{X}$ 中的样本 点按照 RBF 响应值从低到高排列, 等分为 $K$ (例如 取 100)组。根据各组 RBF 响应值的平均值大小及代 理模型复相关系数 $R^{2}$ 构造概率分布模型, 此分布模 型使得 RBF 响应值较小的样本点被选取的概率较 大, 由此每次迭代在 $\boldsymbol{X}$ 中取 $n_{v}$ 个点生成高精度样本 点 ${ }^{[14]}$ 。

图 1 是 MPS 优化六驼峰函数(Six-hump Camelback function, SC)时构造分布模型过程的示意图。 SC 函数表达式为 ${ }^{[17]}$

$$
\begin{gathered}
f(x)=4 x_{1}^{2}-\frac{21}{10} x_{1}^{4}+\frac{1}{3} x_{1}^{6}+x_{1} x_{2}-4 x_{2}^{2}+4 x_{2}^{4} \\
\left(x_{1}, x_{2}\right) \in[-2,2]^{2}
\end{gathered}
$$

图中 $\hat{f}$ 表示简单样本点对应 $\mathrm{RBF}$ 响应值, 从小到大 排列; $g$ 表示 10000 个简单样本点等分为 100 组, 每组 RBF 响应值的均值; $\tilde{g}$ 表示用 $g$ 的最大值减去
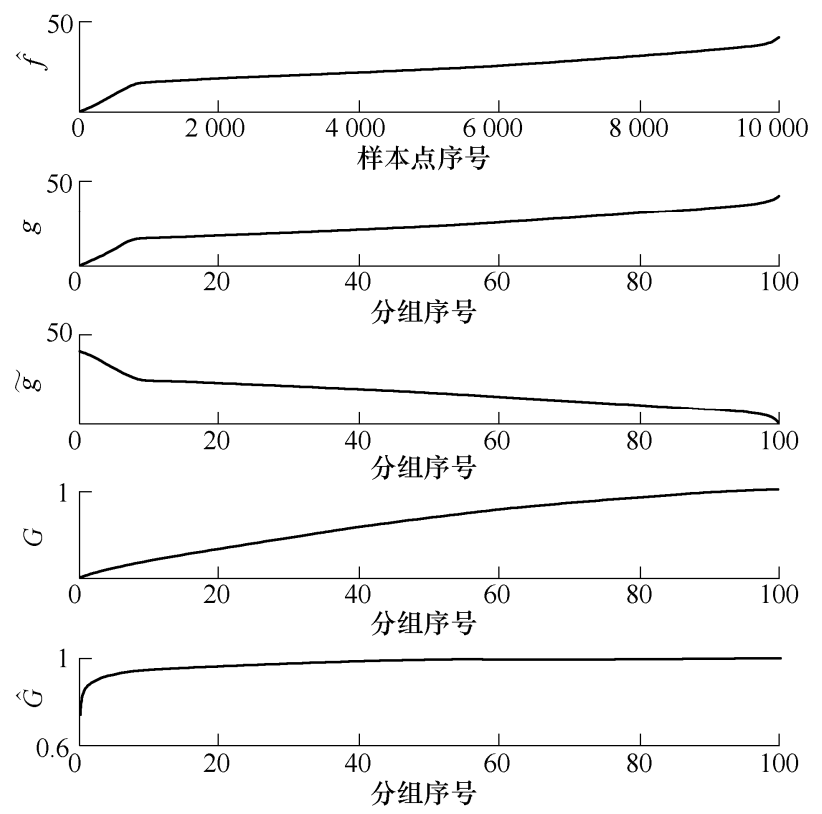

图 1 分布模型示意图
各组的 $g$ 值, 即分布模型的概率密度函数; $G$ 表示 对 $\tilde{g}$ 值积分并归一化的结果，即分布模型的分布函 数; $\hat{G}$ 表示加速因子 $r>1^{[14]}$ 时调整后的分布函数 值, 保证 RBF 响应值较小的样本点组被选取的概率 较大。

MPS 算法选取 $\boldsymbol{Y}$ 中真实模型响应值最小的点 及距离该点最近的 $\left(n_{v}+1\right)\left(n_{v}+2\right) / 2$ 个点, 根据所有 被选中点的取值范围的上下界构造子区间, 并在该 子区间内构造二次响应面, 其表达式为

$$
\tilde{y}=\beta_{0}+\sum_{i=1}^{n_{v}} \beta_{i} X_{i}+\sum_{i=1}^{n_{v}} \beta_{i i} X_{i}+\sum_{i=1}^{n_{v}} \sum_{j>i}^{n_{v}} \beta_{i j} x_{i} x_{j}
$$

待定系数 $\beta_{0}, \beta_{i}, \beta_{i i}, \beta_{i j}$ 由最小二乘法确定

$$
\boldsymbol{\beta}=\left(\boldsymbol{X}_{c} \boldsymbol{X}_{c}^{\mathrm{T}}\right)^{-1} \boldsymbol{X}_{c}^{\mathrm{T}} \boldsymbol{y}_{c}
$$

其中 $\boldsymbol{X}_{c}$ 是与构造样本点相关的设计变量矩阵, $\boldsymbol{y}_{c}$ 是构造样本点真实模型响应值组成的列矢量。

如果该二次响应面的复相关系数 $R^{2}$ 接近于 1 , 则在子区域内新增 $\operatorname{round}\left(n_{v} / 2\right)$ 个高精度样本点, 再 次构造二次响应面。如果复相关系数和局部近似精 度值满足要求, 则用序列二次规划方法优化响应面, 把得到的最优解加入高精度样本点集 $\boldsymbol{Y}$ 。当满足最 优性准则和近似精度准则 ${ }^{[6]}$ 时, 可认为算法收玫。

\section{3 追峰采样方法处理高维问题时的缺陷}

DUAN 等 ${ }^{[16]}$ 对 MPS 及其部分衍生算法的性能 进行了详细探讨, 与 GA 相比, MPS 在处理中低维 问题 $\left(n_{v} \leqslant 10\right)$ 时具有明显的效率优势, 但是求解高 维问题 $\left(n_{v}>10\right)$ 时, MPS 需要大量调用分析模型, 求解效率有待进一步提升。以求解 F16 函数为例, MPS 连续运行 10 次的目标函数调用次数的平均值 为 921 , 且最终得到的最优解平均值 29.786 与理论 最优解 25.875 相差较大 ${ }^{[6]}$ 。

本文研究发现, 在处理高维问题的过程中, MPS 会出现连续多次迭代最优性无法改善的情况, 即陷 入 “平台期”。由图 2 中 MPS 求解 F16 问题的迭代 过程可见, 在算法终止前连续 30 次迭代中目标函数 值保持不变, 导致了算法收玫速度低下和计算成本 极大(目标函数调用 1050 次)。事实上, MPS 陷入 “平台期” 时的当前最优点并非 F16 问题的局部最 优解, “平台期” 现象并非由 MPS 陷入局部最优所 导致, 其真实原因在于 MPS 较弱的局部搜索能力。 因此, 为了提高 MPS 求解高维优化问题的效率, 亟 待进一步增强其局部搜索能力。

作者所提出的基于空间缩减序列采样的 SRBF 算法具有较强的局部搜索能力, 可以在目标函数平 均调用 299 次的情况下快速获得理论最优解 $25.875^{[10]}$ 。SRBF 虽然在处理高维问题时效率往往高 
于 MPS, 但其理论框架不够严谨, 难以确保收玫到 全局最优解。

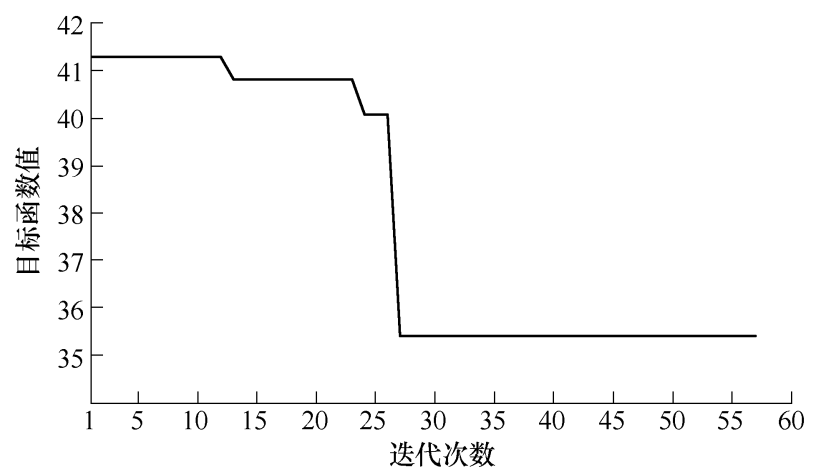

图 2 MPS 求解 F16 问题的目标函数迭代过程

为此, 本文将 SRBF 算法中的核心技术一一重 点设计空间与 MPS 求解框架进行融合, 进一步提升 MPS 的局部搜索能力, 有效折中全局探索和局部搜 索之间的矛盾。

\section{2 快速追峰采样方法}

\section{1 快速追峰采样近似优化策略求解框架}

涉及高精度模型的非线性工程优化问题的数学 模型为

$$
\begin{array}{ll}
\text { find } & \boldsymbol{x}=\left(x_{1}, x_{2}, \cdots, x_{v}\right) \\
\min & f(\boldsymbol{x}) \\
\text { s.t. } & g_{j}(\boldsymbol{x}) \geqslant 0 \quad(j=1,2, \cdots, l), \\
& h_{k}(\boldsymbol{x})=0 \quad(k=1,2, \cdots, m) . \\
& x_{L, i} \leqslant x_{i} \leqslant x_{U, i} \quad\left(i=1,2, \cdots, n_{v}\right)
\end{array}
$$

对于基于代理模型的近似优化策略, 求解的目标函 数和约束条件 $f(\boldsymbol{x}), g_{j}(\boldsymbol{x}), h_{k}(\boldsymbol{x})$ 变为数值近似模 型 $\hat{f}(\boldsymbol{x}), \hat{g}_{j}(\boldsymbol{x}), \hat{h}_{k}(\boldsymbol{x})$ 。

快速追峰采样近似优化策略的具体步骤如下。

(1) 用基于 Maximin 准则的拉丁超方设计进 行初始采样, 在给定的设计空间内生成 $\left(n_{v}+1\right)\left(n_{v}+2\right) / 2-1+n_{v}$ 个高精度样本点, 将初始样 本点及其真实模型响应值加入高精度样本点集 $\boldsymbol{Y}$ 。

（2）用高精度样本点集 $\boldsymbol{Y}$ 中所有点构造 RBF 代 理模型。 $k=1$ 时执行第 4 步, 否则执行第 3 步。

(3) 根据样本点分配策略, 将 $n_{v}$ 个高精度样本 点分为 $n_{G}$ 个全局探索样本点和 $n_{L}$ 个局部探索样本 点。分配依据包括设计变量维度 $n_{v}$ 、目标函数持续 未改善迭代次数 $\chi$ 、当前 $\mathrm{RBF}$ 模型复相关系数 $R^{2}$ 、 上一步迭代中的局部搜索样本点数 $n_{L 0}$ 、局部搜索 持续改善目标函数的迭代次数 $n_{i}$ 。具体步骤参见 2.2 节。

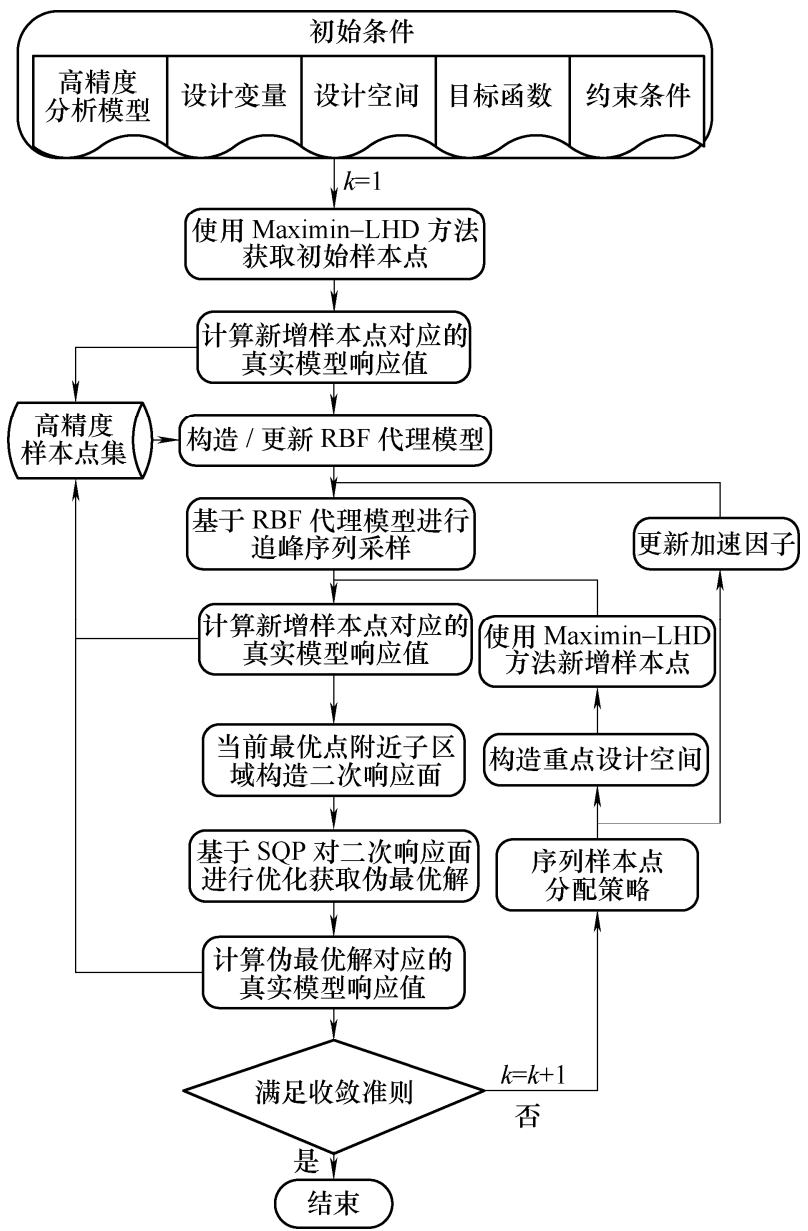

图 3 快速追峰采样近似优化策略(RMPS-SDS)求解框架

（4）设计空间内随机生成 $N$ (本文取 $N=10000$ ) 个简单样本点, 将它们及其 RBF 响应值加入简单样 本点集 $\boldsymbol{X}$ 。用新增样本点的 RBF 响应值生成概率 分布模型进行追峰采样, 每次迭代从 $\boldsymbol{X}$ 中选取 $n_{G}$ 个 样本点(迭代次数 $k=1$ 时, 取 $n_{G}=n_{v}$ ), 将新增样本 点及其真实模型响应值加入高精度样本点集 $\boldsymbol{Y}$ 。

（5）构造重点设计空间进行局部搜索，具体步 骤参见 2.3 节。在重点设计空间内通过基于 Maximin 准则的拉丁超方设计新增 $n_{L}$ 个样本点。

(6) 将 $n_{v}=n_{G}+n_{L}$ 个新增样本点及其真实模型 响应值加入高精度样本点集 $\boldsymbol{Y}$, 选择高精度样本点 集中真实模型响应值最小的点以及与其距离最近的 $\left(n_{v}+1\right)\left(n_{v}+2\right) / 2$ 个点构造二次响应面, 取所有被 选中点各维坐标值的最小值和最大值为子区域的 上下界。

(7) 如果二次响应面的 $R^{2}$ 值接近 1 (例如 $\left.R^{2}>0.9\right)$, 则在子区域内新增 $\operatorname{round}\left(n_{v} / 2\right)$ 个样本 点, 用这 $\left(n_{v}+1\right)\left(n_{v}+2\right) / 2+1+\operatorname{round}\left(n_{v} / 2\right)$ 个点重 新构造二次响应面并计算复相关系数 $R^{2}$ 和局部近 似精度 Diff, 如果满足 $R^{2}>0.9999$ 且 Diff $\leqslant 0.01$, 在子区域内用序列二次规划方法优化二次响应面, 将得到的最优点(伪最优解)及其真实模型响应值加 
入高精度样本点集 $\boldsymbol{Y}$ 。

(8) 当连续两次迭代目标函数改善程度足够小 $\left(\left|f\left(\boldsymbol{x}_{k}^{*}\right)-f\left(\boldsymbol{x}_{k-1}^{*}\right)\right| / f\left(\boldsymbol{x}_{k}^{*}\right) \leqslant \xi_{1}\right.$, 通常取 $\left.\xi_{1} \leqslant 0.001\right)$, 且代理模型在最优点 $x_{k}^{*}$ 处近似精度足够高 $\left(\left|\hat{f}\left(\boldsymbol{x}_{k}^{*}\right)-f\left(\boldsymbol{x}_{k-1}^{*}\right)\right| / f\left(\boldsymbol{x}_{k}^{*}\right) \leqslant \xi_{2}\right.$, 通常取 $\left.\xi_{2} \leqslant 0.01\right)$, 则认为迭代已经收玫, 算法终止。对于高维问题, 通常设定最大迭代次数 $N_{\mathrm{it}}^{\mathrm{max}}$ 或最大模型调用次数 $N_{\mathrm{fe}}^{\max }$ 以控制计算成本。当迭代次数 $N_{\mathrm{it}} \geqslant N_{\mathrm{it}}^{\max }$ 或模 型调用次数 $N_{\mathrm{fe}} \geqslant N_{\mathrm{fe}}^{\max }$ 时, 同样停止迭代。如果不 满足上述收玫准则, 执行第 3 步并更新追峰采样方 法中的加速因子。

\section{2 样本点分配策略}

为有机融合重点设计空间方法与 MPS 框架, 本 文提出了一种样本点分配策略, 主要根据设计变量 维度 $n_{v}$ 、目标函数持续未改善迭代次数 $\chi$ 、局部搜 索持续改善目标函数的迭代次数 $n_{i}$ 、当前 $\mathrm{RBF}$ 模型 复相关系数 $R^{2}$ 以及上一代迭代中的局部搜索样本 点值 $n_{L 0}$ 构造分配算子, 将 $n_{v}$ 个新增样本点分为 $n_{G}$ 个全局探索样本点和 $n_{L}$ 个局部搜索样本点。当迭代 次数 $k=1$ 时, $n_{L 0}=0$; 当迭代次数 $k>1$ 时, $n_{L 0}$ 是 第 $k-1$ 次迭代时的局部搜索样本点数。全局探索样 本点沿用基于概率分布模型的追峰采样机理, 局部 搜索样本点则取自构造的重点设计空间内。样本点 分配策略包括如下步骤。

(1) 统计局部搜索持续改善目标函数的迭代次数 $n_{i}$, 当 $n_{i}>3$ 时, 设局部搜索标记 $f l a g=1$; 否则设 flag $=0$;

（2）当目标函数持续未改善迭代次数 $\chi \geqslant 2$ 或 局部搜索标记 flag $=1$, 且径向基函数复相关系数 $R^{2}>0.9$ 时, 局部搜索样本点数 $n_{L}>0$, 执行步骤 3 ; 否则 $n_{L}=0$ ，执行步骤 4 ;

(3) 如果局部探索样本点个数 $n_{L}>0$, 则取 $n_{L}=\operatorname{round}\left(n_{v} / 2\right)+2 \times(\chi-2)$, 执行步骤 4 ;

(4) 全局探索样本点个数 $n_{G}=n_{v}-n_{L}$, 如果 $n_{G} \leqslant 2$, 则取 $n_{G}=2$, 且 $n_{L}=n_{v}-2$, 样本点分配策 略结束; 如果 $n_{G} \geqslant 2$, 则不修改 $n_{G}$ 和 $n_{L}$ 的数值, 样 本点分配策略结束。

样本点分配策略的伪代码如下。

Algorithm 1. 样本点分配策略

Input: 设计变量维度 $n_{v}$; 目标函数持续未改善迭代 次数 $\chi$; 局部搜索持续改善目标函数的迭代 次数 $n_{i}$; 当前 $\mathrm{RBF}$ 模型复相关系数 $R^{2}$; 上 一步迭代中的局部搜索样本点数 $n_{L 0}$

Output: 全局探索样本点数 $n_{G}$, 局部搜索样本点数 $n_{L}$ 1 if $n_{L 0}>0$ and $\chi=0$ then

$2 \quad n_{i} \leftarrow n_{i}+1$
3 elseif $n_{L 0}>0$ and $\chi>0$ then

4

$n_{i} \leftarrow 0$

flag $\leftarrow 0$

end if

if $n_{i}>3$ then

flag $\leftarrow 1$

end if

if $\chi \geqslant 2$ or flag $=1$ and $R^{2}>0.9$ then

$n_{L} \leftarrow \operatorname{round}\left(n_{L} / 2\right)+2 * \operatorname{abs}(\chi-2)$

if $n_{L}>n_{v}-2$ then

$$
n_{L} \leftarrow n_{v}-2
$$

end if

15 else

16

17 end if

$18 n_{G} \leftarrow n_{v}-n_{L}$

19 return $\left[n_{G}, n_{L}\right]$

\section{3 重点设计空间构造}

重点设计空间(SDS) ${ }^{[10]}$ 是 RMPS-SDS 提高优化 效率和收敛性的关键技术。重点设计空间是当前迭 代可能最优解附近区域, 该区域可能存在全局最优 解。通过逐次增加样本点, 重点设计空间有助于提 高 $\mathrm{RBF}$ 模型在全局最优解附近的近似精度。重点设 计空间的构造方法如下。

(1) 如果当前最优点处目标模型响应值小于上 一次迭代目标模型响应值(如: $\left.y_{k}^{*}<y_{k-1}^{*}\right)$, 或者当前 迭代次数 $k=1$, 则 SDS 的尺寸 $\boldsymbol{B}_{k}$ 由式(10)计算。式 中, 当 $k \geqslant 2$ 时, SDS 尺寸由最近两次迭代最优点之 间的距离确定; 当 $k=1$ 时, SDS 尺寸由当前可能最 优点和初始样本点中最大误差点 $\bar{x}_{\text {max }}$ 之间的距离确 定。样本点处相对误差通过逐一交叉验证(Leave-one out cross validation, LOO-CV $)^{[18]}$ 计算。SDS 的中心 $S_{\text {center }}$ 为当前最优点 $\boldsymbol{x}_{k}^{*}$ 。执行步骤 4 。

$$
\boldsymbol{B}_{k}= \begin{cases}\left\|\boldsymbol{x}_{k}^{*}-\boldsymbol{x}_{k-1}^{*}\right\| & k \geqslant 2 \\ \left\|\boldsymbol{x}_{k}^{*}-\overline{\boldsymbol{x}}_{\text {max }}\right\| & k=1\end{cases}
$$

（2）如果当前最优解大于上一次迭代可能最优 解(如 $y_{k}^{*} \geqslant y_{k-1}^{*}$ ), 由 LOO-CV 计算当前可能最优点 处相对误差 $\varepsilon_{k}$ 。

(3) 如果 $\varepsilon_{k}<\varepsilon_{k-1}$, 则增加当前迭代 SDS 的尺寸 为 $\boldsymbol{B}_{k} \cdot \alpha$, 其中 SDS 尺寸调节系数 $\alpha$ 是大于 1 的常数。 如果 $\varepsilon_{k} \geqslant \varepsilon_{k-1}$, 则减小当前迭代 SDS 的尺寸为 $\boldsymbol{B}_{k} / \alpha$ 。

(4) 判断当前 SDS 每一维尺寸是否小于最小设 计空间尺寸系数(如 $\boldsymbol{B}_{k}<\sigma\left|\boldsymbol{S}_{0}\right|$ ), 如果是, 则修改该 维空间尺寸为 $\sigma\left|\boldsymbol{S}_{0}\right|$ 。其中 $\sigma$ 称为最小尺寸系数。 
(5) 当前可能 SDS 用符号 $\boldsymbol{S}_{k}^{\mathrm{t}}=\left[\boldsymbol{B}_{k}^{(\mathrm{L})}, \boldsymbol{B}_{k}^{(\mathrm{U})}\right]$ 表示, $\boldsymbol{B}_{k}^{(\mathrm{L})}$ 和 $\boldsymbol{B}_{k}^{(\mathrm{U})}$ 分别表示 SDS 的下边界和上边界, 由式 (11)计算。为保证 SDS 不超出初始设计空间, 第 $k$ 次 迭代 SDS 尺寸为 $\boldsymbol{S}_{k}^{t}$ 和 $\boldsymbol{S}_{0}$ 之间的交集, $\boldsymbol{S}_{k}=\boldsymbol{S}_{k}^{t} \cap \boldsymbol{S}_{0}$ 。

$$
\begin{aligned}
& \boldsymbol{B}_{k}^{(\mathrm{L})}=S_{\text {center }}-\boldsymbol{B}_{k} \\
& \boldsymbol{B}_{k}^{(\mathrm{U})}=S_{\text {center }}+\boldsymbol{B}_{k}
\end{aligned}
$$

需要注意的是, 根据构造方法, 重点设计空间 一般需要 3 次迭代的信息, 为了避免额外增加算法 开销, 充分利用 MPS 算法框架得到的高精度样本点 信息, 在每一次迭代过程中都构造重点设计空间, 但当 MPS 的局部搜索能力满足要求时不分配局部搜 索样本点。

\section{3 算法性能测试}

为测试 RMPS-SDS 处理高维问题时的效率, 本 文选择了 10 个中高维数值测试问题和 2 个高维工程 优化案例, 并将其中的目标函数视为高精度分析模 型, 选取标准的追峰采样方法(MPS)和遗传算法 (Genetic algorithm, GA)作为对照。对三种算法都设 置最大目标函数调用次数 $N_{\mathrm{fe}}^{\text {max }}$, 即当模型调用次数 大于 $N_{\mathrm{fe}}^{\max }$ 时终止迭代, 将当前最好结果作为最优 解。为排除随机因素影响, 每种算法对各测试算例 分别连续优化 10 次, 统计优化结果进行对比分析。

\section{1 算法参数设置}

MPS 与 RMPS-SDS 的算法参数设置如表 1 所示。

\begin{tabular}{|c|c|c|}
\hline 可调节参数 & MPS 取值 & RMPS-SDS 取值 \\
\hline $\begin{array}{l}\text { 初始高精度 } \\
\text { 样本点数 }\end{array}$ & $\left(n_{v}+1\right)\left(n_{v}+2\right) / 2-1+n_{v}$ & $\left(n_{v}+1\right)\left(n_{v}+2\right) / 2-1+n_{v}$ \\
\hline $\begin{array}{l}\text { 每次迭代简单 } \\
\text { 样本点数 }\end{array}$ & $1 \times 10^{4}$ & $1 \times 10^{4}$ \\
\hline $\begin{array}{l}\text { 子区域新增 } \\
\text { 样本点条件 }\end{array}$ & $R^{2}>0.9$ & $R^{2}>0.9$ \\
\hline 子区域 & $R^{2}>0.9999$ & $R^{2}>0.9999$ \\
\hline 局部优化条件 & Diff $\leqslant 0.1$ & Diff $\leqslant 0.1$ \\
\hline $\begin{array}{c}\text { 目标函数 } \\
\text { 改善程度容差 }\end{array}$ & $\xi_{1} \leqslant 0.001$ & $\xi_{1} \leqslant 0.001$ \\
\hline $\begin{array}{c}\text { 近似精度 } \\
\text { 容差 }\end{array}$ & $\xi_{2} \leqslant 0.01$ & $\xi_{2} \leqslant 0.01$ \\
\hline $\begin{array}{c}\text { 最大目标函数 } \\
\text { 调用次数 }\end{array}$ & $N_{\mathrm{fe}} \geqslant N_{\mathrm{fe}}^{\max }=5000$ & $N_{f e} \geqslant N_{f e}^{\max }=5000$ \\
\hline $\begin{array}{l}\text { SDS 尺寸 } \\
\text { 调节系数 } \alpha\end{array}$ & - & 2 \\
\hline $\begin{array}{l}\text { SDS 最小 } \\
\text { 尺寸系数 } \sigma\end{array}$ & - & 0.05 \\
\hline
\end{tabular}

\section{表 1 MPS 与 RMPS-SDS 算法参数设置}

GA 算法直接使用 Matlab 的 ga 工具包, 相关参 数取默认值。为了便于对比研究, 根据 MPS 与
RMPS-SDS 测试问题优化结果, 设置 GA 优化各测 试问题时的 $N_{\mathrm{fe}}^{\max }$ 如表 2 所示。

表 2 GA 算法 $N_{\mathrm{fe}}^{\max }$ 设置

\begin{tabular}{ccccccc}
\hline 测试函数 & HN & F16 & R10 & R20 & SUR10 & SUR20 \\
\hline$N_{\mathrm{fe}}^{\max }$ & 300 & 1100 & 5000 & 5000 & 5000 & 5000 \\
\hline 测试函数 & ZF10 & ZF20 & GR10 & GR20 & WW10 & AS10 \\
\hline$N_{\mathrm{fe}}^{\max }$ & 5000 & 5000 & 200 & 400 & 400 & 100 \\
\hline
\end{tabular}

\section{2 测试问题描述}

\subsection{1 数值测试问题}

Hartman 函数 $\left(\mathrm{HN}, n_{v}=6\right)^{[14]}$

$$
\begin{gathered}
f(\boldsymbol{x})=-\sum_{i=1}^{n_{v}} c_{i} \exp \left[-\sum_{j=1}^{n_{v}} \alpha_{i j}\left(x_{j}-p_{i j}\right)^{2}\right] \\
x \in[0,1] \quad i=1,2, \cdots, n_{v}
\end{gathered}
$$

其中 $\alpha_{i j}, c_{i}, p_{i j}$ 取值见文献[14]。

16 维函数 $\left(\mathrm{F} 16, n_{v}=16\right)^{[14]}$

$$
\begin{gathered}
f(\boldsymbol{x})=\sum_{i=1}^{16} \sum_{j=1}^{16} a_{i j}\left(x_{i}^{2}+x_{i}+1\right)\left(x_{j}^{2}+x_{j}+1\right) \\
i, j=1,2, \cdots, 16 \quad x_{k} \in[-1,1] \\
k=1,2, \cdots, n_{v}
\end{gathered}
$$

其中 $a_{i j}$ 取值见文献[14]。

10 维、 20 维 Rosenbrock 函数(R10/R20, $n_{v}=10 /$ $\left.n_{v}=20\right)^{[16]}$

$$
\begin{gathered}
f(\boldsymbol{x})=\sum_{i=1}^{n_{v}-1}\left(100\left(x_{i+1}-x_{i}^{2}\right)^{2}+\left(x_{i}-1\right)^{2}\right) \\
\boldsymbol{x} \in[-5,5] \quad i=1,2, \cdots, n_{v}
\end{gathered}
$$

10 维、 20 维 SUR-T1-14 函数(SUR10/SUR20, $\left.n_{v}=10 / n_{v}=20\right)^{[19]}$

$$
\begin{gathered}
f(\boldsymbol{x})=\left(x_{1}-1\right)^{2}+\left(x_{n_{v}}-1\right)^{2}+n_{v} \sum_{i=1}^{n_{v}-1}\left(n_{v}-i\right)\left(x_{i}^{2}-x_{i+1}\right)^{2} \\
x_{i} \in[-3,2] \quad i=1,2, \cdots, n_{v}
\end{gathered}
$$

10 维、 20 维 Zakharov 函数 (ZF10/ZF20, $n_{v}=10$ / $\left.n_{v}=20\right)^{[8]}$

$$
\begin{gathered}
f(\boldsymbol{x})=\sum_{i=1}^{n_{v}} x_{i}^{2}+\left(\sum_{i=1}^{n_{v}} 0.5 i x_{i}\right)^{2}+\left(\sum_{i=1}^{n_{v}} 0.5 i x_{i}\right)^{4} \\
x_{i} \in[-5,10] \quad i=1,2, \cdots, n_{v}
\end{gathered}
$$

10 维、 20 维 Griewank 函数(GR10/GR20, $n_{v}=10$ / $\left.n_{v}=20\right)^{[8]}$

$$
\begin{gathered}
f(\boldsymbol{x})=\sum_{i=1}^{n_{v}} \frac{x_{i}^{2}}{4000}-\prod_{i=1}^{n_{v}} \cos \left(\frac{x_{i}}{\sqrt{i}}\right)+1 \\
x_{i} \in[-600,600] \quad i=1,2, \cdots, n_{v}
\end{gathered}
$$

3.2.2 机翼重量优化问题(WW10)

10 维机翼重量优化算例 ${ }^{[20]}$ 中, 机翼重量的经验 
求解公式如下

$$
\begin{gathered}
W=0.036 x_{1}^{0.0758} x_{2}^{0.0035}\left(\frac{x_{3}}{\cos ^{2} x_{4}}\right)^{0.6} x_{5}^{0.006} x_{6}^{0.04} . \\
\left(\frac{100 x_{7}}{\cos x_{4}}\right)^{-0.3}\left(x_{8} x_{9}\right)^{0.49}+x_{1} x_{10}
\end{gathered}
$$

式中, $x_{1}, x_{2}, \cdots, x_{10}$ 对应的物理含义及取值范围如表 3 所示。表 3 中的基准值来自塞斯纳 C172 “天鹰” 小型飞机, 相应的各设计变量取值在基准量上下浮 动适当范围作为设计空间上下界。

\section{表 310 维机翼重量优化问题参数表}

\begin{tabular}{lccc}
\hline \multicolumn{1}{c}{ 设计变量 } & 基准值 & 下界 & 上界 \\
\hline 翼面积 $x_{1} / \mathrm{ft}^{2}$ & 174 & 150 & 200 \\
机翼携带燃油重量 $x_{2} / \mathrm{lb}$ & 252 & 220 & 300 \\
展弦比 $x_{3}$ & 7.52 & 6 & 10 \\
后掠角 $x_{4} /\left(^{\circ}\right)$ & 0 & -10 & 10 \\
巡航动压 $x_{5} /\left(\mathrm{lb} / \mathrm{ft}^{2}\right)$ & 34 & 16 & 45 \\
跟梢比 $x_{6}$ & 0.672 & 0.5 & 1 \\
翼型相对厚度 $x_{7}$ & 0.12 & 0.08 & 0.18 \\
极限过载 $x_{8}$ & 3.8 & 2.5 & 6 \\
全机设计重量 $x_{9} / \mathrm{lb}$ & 2000 & 1700 & 2500 \\
单位面积涂料重量 $x_{10} /\left(\mathrm{lb} / \mathrm{ft}^{2}\right)$ & 0.064 & 0.025 & 0.08 \\
\hline
\end{tabular}

\subsection{3 翼型气动优化问题(AS10)}

选用文献[21]中的 10 维翼型气动优化问题进一 步检验 RMPS-SDS 的工程实用性。

选用 Hicks-Henne 形函数 ${ }^{[22]}$ 描述翼型的几 何外形。采用 C-H 型结构化网格, 为保证计算 精度, 在翼面附近加大网格密度。采用 Fluent 作为翼型流场解算器, 以 N-S 方程为主控方程, 湍流模型为 Spalart-Allmaras (S-A)模型。使用实验值 校验 CFD 模型的分析精度, $M a=0.63$, 攻角 $\alpha=2^{\circ}$ 时 NACA0012 翼型压力系数分布的风洞实验值与
计算值如图 4 所示, 结果验证了 CFD 模型的合 理性。

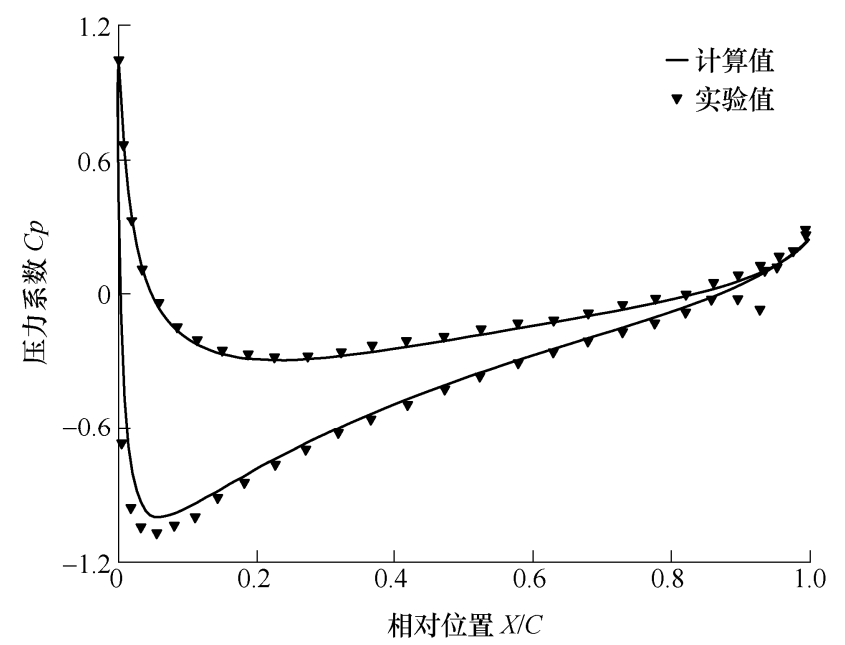

图 4 NACA0012 压力分布实验值与计算值对比

以 NACA0012 为基准翼型, 优化翼型在 $M a=0.63$, 攻角 $\alpha=2^{\circ}$ 工况下的升阻比, 约束条件 为翼型的最大相对厚度不超过基准翼型的 $80 \%$ 。优 化模型如下

$$
\begin{array}{ll}
\max & f(\boldsymbol{x})=C l / C d \\
\text { s.t. } & t_{\max } \geqslant 0.8 \cdot t_{\max }^{0} \\
& x_{i} \in[-0.01,0.01], \quad i=1,2, \cdots, 10
\end{array}
$$

其中 $C d$ 和 $C l$ 分别表示翼型的阻力系数和升力系 数, $t_{\text {max }}$ 表示翼型的最大相对厚度, $t_{\text {max }}^{0}$ 表示 NACA0012 翼型的最大相对厚度。10个设计变量分 别描述沿弦线均匀分布的 5 个控制点处翼型上下表 面的形变量。

\begin{tabular}{|c|c|c|c|c|c|c|c|c|c|c|}
\hline \multirow{3}{*}{$\begin{array}{l}\text { 测试 } \\
\text { 函数 }\end{array}$} & \multirow{3}{*}{$\begin{array}{c}\text { 理论 } \\
\text { 最优解 }\end{array}$} & \multicolumn{3}{|c|}{ MPS } & \multicolumn{3}{|c|}{ RMPS-SDS } & \multicolumn{3}{|c|}{ GA } \\
\hline & & 调用次数 & 最优解 & 最优解 & 调用次数 & 最优解 & 最优解 & 调用次数 & 最优解 & 最优解 \\
\hline & & 均值 & 均值 & 标准差 & 均值 & 均值 & 标准差 & 均值 & 均值 & 标准差 \\
\hline HN & -3.322 & 245 & -2.49 & 0.40 & 236 & -3.32 & 0.056 & 300 & -2.36 & 0.36 \\
\hline F16 & 25.875 & 1054 & 37.87 & 6.93 & 1049 & 25.875 & $9.54 \times 10^{-4}$ & 1100 & 28.96 & 0.65 \\
\hline R10 & 0 & 4902 & 229.5 & 177.8 & 5005 & 9.864 & 1.49 & 5000 & 18.12 & 24.28 \\
\hline R20 & 0 & 4991 & $3.315 \times 10^{4}$ & $3.49 \times 10^{4}$ & 5016 & 39.50 & 4.34 & 5000 & 176.70 & 76.13 \\
\hline SUR10 & 0 & 4921 & 20.11 & 15.1 & 5006 & 1.89 & 1.03 & 5000 & 2.23 & 1.20 \\
\hline SUR20 & 0 & 5000 & 6765 & 6476 & 5015 & 21.04 & 8.20 & 5000 & 185.52 & 64.65 \\
\hline ZF10 & 0 & 4994 & $2.73 \times 10^{6}$ & $1.65 \times 10^{6}$ & 5006 & 0.14 & 0.043 & 5000 & 1.84 & 1.64 \\
\hline ZF20 & 0 & 5000 & $2.36 \times 10^{11}$ & $2.31 \times 10^{11}$ & 5009 & 21.04 & 15.15 & 5000 & 46.50 & 40.27 \\
\hline GR10 & 0 & 157 & 91.54 & 32.66 & 77 & $5.44 \times 10^{-3}$ & 0.054 & 200 & 0.72 & 0.17 \\
\hline GR20 & 0 & 392 & 274.33 & 34.96 & 385 & $1.04 \times 10^{-6}$ & $5.29 \times 10^{-7}$ & 400 & 0.76 & 0.12 \\
\hline WW10 & N/A & 377 & 152.04 & 7.96 & 376 & 123.29 & $6.24 \times 10^{-2}$ & 400 & 145.95 & 9.11 \\
\hline AS10 & N/A & 107 & 46.2877 & 1.9528 & 107 & 49.0653 & 0.6480 & 110 & 46.0365 & 3.1411 \\
\hline
\end{tabular}

\section{3 优化结果}

MPS, RMPS-SDS, GA 三种算法对上述测试问 题的优化结果如表 4 所示, MPS 对 R10/R20、SUR10/

表 4 MPS, RMPS-SDS, GA 的测试问题优化结果 
SUR20、ZF10/ZF20 等算例的优化结果引自文献[8]。 其中, AS10 问题使得目标函数最大, 其余问题均使 得目标函数最小。

对于中维问题 $\mathrm{HN}\left(n_{v}=6\right)$, RMPS-SDS 能在很 少的目标函数调用次数下找到理论全局最优解 -3.32, 而 MPS 和 GA 优化结果偏离理论全局最优 解较远, 且鲁棒性较差。RMPS-SDS 在 F16 问题上 表现出色, 调用目标函数 1049 次左右即可收玫到理 论全局最优解 25.875。由图 5 可以看出, RMPS-SDS 有效缓解了 MPS 中的 “平台期” 现象。特别是第 15 次迭代后, 每一次迭代都能使目标函数值有效 下降。

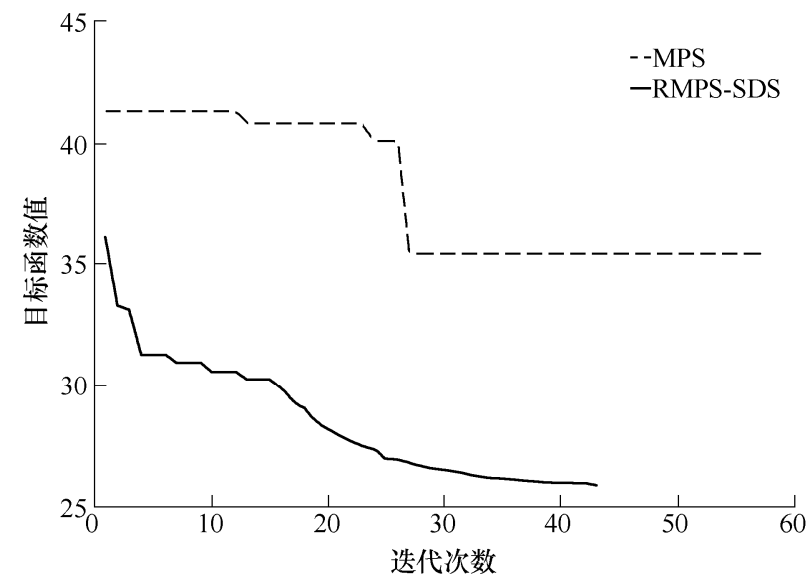

图 5 RMPS-SDS 与 MPS 求解 F16 问题的 目标函数迭代过程对比

Rosenbrock 函数的全局最优点位于一个 “香蕉 形山谷” 内, MPS 在处理这类问题时能力很差, 20 维问题求出的最优解达 $10^{4}$ 量级, 相比之下 RMPS-SDS 的结果明显改善, 且优于 GA 得到的结 果。同样, RMPS-SDS 在处理 SUR 问题时得到的最 优解远远小于 MPS 得到的结果, 且与 GA 相比优势 明显, 特别是 20 维 SUR 问题。ZF 函数只有一个局 部最优点作为全局最优点, 除全局最优点附近外梯 度都极大，优化结果表明 MPS 未能找到全局最优 点附近区域, 而 RMPS-SDS 能得到很好的优化结果。 GR 函数有非常多的局部最优点, 这导致 MPS 难以 给出令人满意的结果。对于 GR10 问题, RMPS-SDS 能在极少的目标函数调用次数下收敛到 $1 \times 10^{-3}$ 量 级, 同时具有较高的鲁棒性。对于 GR20 问题, RMPS-SDS 优化结果与 MPS 和 GA 相比, 在最优性 和鲁棒性上都至少有 $4 \sim 5$ 个数量级的优势。

对于工程设计问题 WW10, RMPS-SDS 平均调 用目标函数 376 次, 得到优化后的机翼重量均值为 $123.29 \mathrm{lb}$, 标准差仅 $6.24 \times 10^{-2}$, 而 MPS 和 GA 分别 调用目标函数 377 和 400 次, 得到优化后的机翼重
量均值分别为 $152.04 \mathrm{lb}$ 和 $145.95 \mathrm{lb}$, 标准差比 RMPS-SDS 结果高两个数量级。RMPS-SDS 同样在 结果最优性和鲁棒性上超过 MPS 和 GA。

对于工程设计问题 AS10, 三种算法所得优化翼 型与初始翼型的对比如表 5 所示。由表中数据可知, RMPS-SDS、MPS、GA 三种算法所得优化翼型最大 相对厚度均满足约束, 且升阻比相对初始翼型分别 提升 $45.24 \% 、 37.02 \% 、 36.27 \%$ ，其中 RMPS-SDS 优化翼型的升阻比提升最为显著，同时约束条件接 近主动约束。根据表 4 数据, RMPS-SDS 优化结果 的标准差仅为 0.6480 , 小于 MPS 和 GA 优化结果 的标准差。因此, RMPS-SDS 在最优性和鲁棒性上 具有较明显的优势，表明 RMPS-SDS 具有良好的工 程实用性。

表 5 翼型气动优化问题优化结果对比

\begin{tabular}{ccccc}
\hline 参数 & 初始翼型 & $\begin{array}{c}\text { RMPS-SDS } \\
\text { 优化翼型 }\end{array}$ & $\begin{array}{c}\text { MPS } \\
\text { 优化翼型 }\end{array}$ & $\begin{array}{c}\text { GA } \\
\text { 优化翼型 }\end{array}$ \\
\hline $\mathrm{Cl}^{*}$ & 0.2254 & 0.4651 & 0.4639 & 0.4523 \\
$\mathrm{Cl}^{*} / C d$ & 33.7826 & 49.0653 & 46.2877 & 46.0365 \\
$t_{\max }^{*}$ & 0.1200 & 0.0996 & 0.1062 & 0.1264 \\
\hline
\end{tabular}

\section{4 结论}

本文提出的算法通过引入重点设计空间方法, 提高了 MPS 算法处理高维问题时的效率。根据本文 提出的样本点分配策略, 每次迭代的新增序列样本 点被分为全局探索样本点和局部搜索样本点, 在重 点设计空间内生成的局部搜索样本点有针对性地提 高了 MPS 算法的局部搜索性能,有利于求解高维优 化问题。数值测试算例与工程设计问题优化结果表 明, RMPS-SDS 与经典的 MPS 和 GA 相比, 在求解 的最优性和鲁棒性方面具有明显的优势。

未来将进一步探索更加高效的样本点自适应分 配策略，并将本文提出的算法应用到更多复杂系统 工程设计中。

\section{参 考 文 献}

[1] SIMPSON $\mathrm{T}$ W, BOOKE A J, GHOSH D, et al. Approximation methods in multidisciplinary analysis and optimization : a panel discussion[J]. Structural and Multidisciplinary Optimization, 2004, 27(5): 302-313.

[2] WANG G G, SHAN S. Review of metamodeling techniques in support of engineering design optimization[J]. Journal of Mechanical Design, 2007, 129(4): 370-380.

[3] MYERS R H, MONTGOMERY D C, ANDERSON- 
COOK C M. Response surface methodology: process and product optimization using designed experiments[D]. New Jersey: John Wiley \& Sons, 2016.

[4] BUHMANN M D. Radial basis functions: theory and implementations[D]. Cambridge: Cambridge University Press, 2003.

[5] SIMPSON T W, MAUERY T M, KORTE J J, et al. Kriging models for global approximation in simulation-based multidisciplinary design optimization[J]. AIAA Journal, 2001, 39(12): 2233-2241.

[6] 龙腾, 刘建, WANG G G, 等. 基于计算试验设计与代 理模型的飞行器近似优化策略探讨 [J]. 机械工程学报, 2016, 52(14): 79-105.

LONG Teng, LIU Jian, WANG G G, et al. Discuss on approximate optimization strategies using design of computer experiments and metamodels for flight vehicle design[J]. Journal of Mechanical Engineering, 2016, 52(14): 79-105.

[7] 龙腾, 郭晓松, 彭否, 等. 基于信赖域的动态径向基函 数代理模型优化策略 [J]. 机械工程学报, 2014, 50(7): 184-190.

LONG Teng, GUO Xiaosong, PENG Lei, et al. Optimization strategy using dynamic radial basis function metamodel based on trust region[J]. Journal of Mechanical Engineering, 2014, 50(7): 184-190.

[8] CHENG G H, YOUNIS A, HAJIKOLAEI K H, et al. Trust region based mode pursuing sampling method for global optimization of high dimensional design problems[J]. Journal of Mechanical Design, 2015， 137(2): 021407.

[9] WANG G G, DONG Z, AITCHISON P. Adaptive response surface method - a global optimization scheme for approximation-based design problems[J]. Engineering Optimization, 2001, 33(6): 707-733.

[10] PENG Lei， LIU Li， LONG Teng. Study of sequential radial basis function for computation-intensive design optimization problem[C]// 12th AIAA Aviation Technology, Integration and Operations (ATIO) Conference, 11st September, 2012, Indianapolis, Indiana. Reston, Virginia: American Institute of Aeronautics and Astronautics, 2012: 5689.

[11] PENG Lei, LIU Li, LONG Teng, et al. An efficient truss structure optimization framework based on CAD/CAE integration and sequential radial basis function metamodel [J]. Structural and Multidisciplinary Optimization, 2014, 50(2): 329-346.

[12] LONG Teng, WU Di, GUO Xiaosong, et al. Efficient adaptive response surface method using intelligent space exploration strategy[J]. Structural and Multidisciplinary Optimization, 2015, 51(6): 1335-1362.
[13] JONES D R, SCHONLAU M, WELCH W J. Efficient global optimization of expensive black-box functions[J]. Journal of Global Optimization, 1998, 13: 455-492.

[14] WANG L, SHAN S, WANG G G. Mode-pursuing sampling method for global optimization on expensive black-box functions[J]. Engineering Optimization, 2004, 36(4): 419-438.

[15] LI Yulin, LIU Li, LONG Teng, et al. Metamodel-based global optimization using fuzzy clustering for design space reduction[J]. Chinese Journal of Mechanical Engineering, 2013, 26(5): 928-939.

[16] DUAN X, WANG G G, KANG X, et al. Performance study of mode-pursuing sampling method[J]. Engineering Optimization, 2009, 41(1): 1-21.

[17] BRANIN F H, HOO S K. A method for finding multiple extrema of a function of $\mathrm{n}$ variables[J]. Numerical Methods, 1972: 231-237.

[18] QUEIPO N V, HAFTKA R T, SHYY W, et al. Surrogate-based analysis and optimization[J]. Progress in Aerospace Sciences, 2005, 41(1): 1-28.

[19] SCHITTKOWSKI K. More test examples for nonlinear programming codes[D]. New York: Springer Science \& Business Media, 2012.

[20] FORRESTER A, SOBESTER A, KEANE A. Engineering design via surrogate modelling: a practical guide[D]. Chichester: John Wiley \& Sons, 2008.

[21] 龙腾, 刘莉, 孟令涛, 等. 基于代理模型的翼型多目标集 成优化设计[J]. 系统仿真学报, 2010, 22(07): 1604-1608. LONG Teng, LIU Li, MENG Lingtao, et al. Multi-objective integrated optimization of airfoil based on surrogate model[J]. Journal of System Integration, 2010, 22(07): 1604-1608.

[22] KHURANA M, WINARTO H, SINHA A. Airfoil geometry parameterization through shape optimizer and computational fluid dynamics[C]// 46th AIAA Aerospace Sciences Meeting and Exhibit, 10th January, 2008, Reno, Nevada. Reston, Virginia: American Institute of Aeronautics and Astronautics, 2008: 295.

作者简介: 武宇飞, 男, 1996 年出生, 博士研究生。主要研究方向为 飞行器总体设计、多学科优化理论与应用。

Email: wuyufei@bit.edu.cn

龙腾(通信作者), 男, 1982 年出生, 博士, 教授, 博士研究生导师。主 要研究方向为飞行器总体设计、多学科优化理论与应用、飞行器协同 控制与决策。

Email: tenglong@bit.edu.cn; bitryu@gmail.com

史人赫, 男, 1990 年出生, 博士研究生。主要研究方向为飞行器总体 设计、多学科优化理论与应用。

Email: shirenhe@bit.edu.cn

WANG G Gary, 男, 1971 年出生, 博士, 教授, 博士研究生导师。主 要研究方向为复杂系统设计优化、工程设计优化。

E-mail: gary_wang@sfu.ca 